\title{
Propagation of Partially Coherent and Partially Polarized Beams Through Periodic Linear Deterministic Structures.
}

\author{
Juan Carlos González de Sande \\ ETSIS de Telecomunicación \\ Universidad Politécnica de Madrid \\ Campus Sur 28031 Madrid, Spain \\ Email: jcgsande@ics.upm.es
}

\author{
Gemma Piquero \\ Departamento de Óptica \\ Universidad Complutense de Madrid \\ Ciudad Universitaria 28040 Madrid, Spain \\ Email: piquero@ucm.es
}

\author{
Massimo Santarsiero \\ Dipartimento di Ingegneria \\ Università Roma Tre and CNISM \\ Via V. Volterra 62, 00146 Rome, Italy \\ Email: msantarsiero@uniroma3.it
}

\begin{abstract}
The propagation of partially coherent and partially polarized beams after a linear deterministic periodic structure is investigated. Spatial irradiance, degree of polarization and state of polarization across the transverse plane are obtained at the exit plane of the structure as well as after paraxial propagation beyond it.
\end{abstract}

\section{Introduction}

There are a lot of optical set ups in which diffraction gratings are used. Generally, this kind of elements are studied within the scalar theory of light and assuming completely coherent light [1], [2]. The influence of the lack of coherency has been considered for the far field [3], [4] and small shift from the Bragg condition as well as broadening have been predicted. Recently, it has been shown that when the vectorial nature of light is taken into account, different and surprising results are obtained [3], [5], [6]. In this work we consider the propagation of partially coherent and partially polarized beams through and after a generic periodic linear deterministic structure (PLDS), i. e., a periodic structure that can be represented by a Jones matrix. Analytical expression of the propagated beam after the PLDS is given. As a particular example of PLDS, a sinusoidal amplitude grating is analyzed in detail. Different effects on the polarization pattern are obtained for a radially polarized input beam.

\section{Theory}

Under paraxial propagation conditions, coherence and polarization characteristics of a quasi-monochromatic light beam can be described by means of its $2 \times 2$ Beam Coherence-Polarization (BCP) matrix. Assuming that the beam propagates along the $z$ direction, the elements of the BCP matrix are defined as [7], [8]

$$
J_{j k}\left(\mathbf{r}_{1}, \mathbf{r}_{2}, z\right)=\left\langle E_{j}\left(\mathbf{r}_{1}, z ; t\right) E_{k}^{*}\left(\mathbf{r}_{2}, z ; t\right)\right\rangle,
$$

where $E_{j}(\mathbf{r}, z ; t)$, with $j, k=x, y$, are the Cartesian components of the time-dependent electric field and $\mathbf{r}$ is the position vector in a plane perpendicular to the $z$ direction. The angle brackets represent temporal average and the asterisk denotes conjugation.
By evaluating the BCP matrix at coincident points, $\mathbf{r}_{1}=$ $\mathbf{r}_{2}=\mathbf{r}$, the irradiance, the degree of polarization (DoP) and the Stokes vector can be obtained as [9], [10]

$$
\begin{gathered}
I(\mathbf{r}, z)=\operatorname{Tr}\{\widehat{J}(\mathbf{r}, \mathbf{r}, z)\} \\
p(\mathbf{r}, z)=\sqrt{1-\frac{4 \operatorname{Det}\{\widehat{J}(\mathbf{r}, \mathbf{r}, z)\}}{\operatorname{Tr}^{2}\{\widehat{J}(\mathbf{r}, \mathbf{r}, z)\}}} \\
\mathbf{S}(\mathbf{r})=\left(\begin{array}{c}
S_{0}(\mathbf{r}, z) \\
S_{1}(\mathbf{r}, z) \\
S_{2}(\mathbf{r}, z) \\
S_{3}(\mathbf{r}, z)
\end{array}\right)=\left(\begin{array}{c}
J_{x x}(\mathbf{r}, \mathbf{r}, z)+J_{y y}(\mathbf{r}, \mathbf{r}, z) \\
J_{x x}(\mathbf{r}, \mathbf{r}, z)-J_{y y}(\mathbf{r}, \mathbf{r}, z) \\
2 \operatorname{Re}\left\{J_{y x}(\mathbf{r}, \mathbf{r}, z)\right\} \\
2 \operatorname{Im}\left\{J_{y x}(\mathbf{r}, \mathbf{r}, z)\right\}
\end{array}\right)
\end{gathered}
$$

respectively. Note that $I(\mathbf{r}, z)=S_{0}(\mathbf{r}, z)$.

A deterministic linear optical element with negligible thickness, placed at $z=0$ plane, transforms the BCP matrix of an incoming field according to the following rule [8]:

$$
\widehat{J}\left(\mathbf{r}_{1}, \mathbf{r}_{2}, 0\right)=\widehat{T}\left(\mathbf{r}_{1}\right) \widehat{J}^{\mathrm{in}}\left(\mathbf{r}_{1}, \mathbf{r}_{2}, 0\right) \widehat{T}^{\dagger}\left(\mathbf{r}_{2}\right),
$$

where $\widehat{T}(\mathbf{r})$ is the Jones matrix of the optical element, $\widehat{J}^{\mathrm{in}}$ is the BCP matrix of the field across the input plane of the element, and the dagger denotes Hermitian conjugation.

For a PLDS, the elements of the Jones matrix can be expressed as a Fourier expansion, that is,

$$
T_{j k}(\mathbf{r})=\sum_{q} a_{j k}^{q} \exp \left(\mathrm{i} \gamma_{j k}^{q} \cdot \mathbf{r}\right)
$$

where $a_{j k}^{q}$ are complex coefficients and the vectors $\gamma_{j k}^{q}$ are reciprocal lattice vectors. Amplitude and phase gratings, polarization gratings [11], and double-wedge depolarizers [12] are examples of this class of elements.

\section{Propagation after a periodic structure}

The BCP matrix across any transversal plane beyond a linear deterministic periodic structure can be calculated if the $\mathrm{BCP}$ is known across the exit plane. In particular, the following expression can be used for the free propagation under paraxial conditions [9], [13]:

$$
\begin{aligned}
& J_{j k}\left(\mathbf{r}_{1}, \mathbf{r}_{2}, z\right)= \\
& \quad \iint K_{z}^{*}\left(\mathbf{r}_{1}, \boldsymbol{\rho}_{1}\right) J_{j k}\left(\boldsymbol{\rho}_{1}, \boldsymbol{\rho}_{2}, 0\right) K_{z}\left(\mathbf{r}_{2}, \boldsymbol{\rho}_{2}\right) d^{2} \rho_{1} d^{2} \rho_{2} .
\end{aligned}
$$


where $\rho$ is the position vector across the $z=0$ plane and

$$
K_{z}(\mathbf{r}, \boldsymbol{\rho})=\frac{-\mathrm{i}}{\lambda z} \exp \left[\frac{\mathrm{i} 2 \pi z}{\lambda}+\frac{\mathrm{i} \pi}{\lambda z}(\mathbf{r}-\boldsymbol{\rho})^{2}\right]
$$

is the direct paraxial propagator and $\lambda$ the wavelength.

On substituting from Eq. (8) into Eq. (7) and using Eq. (5), the elements of the BCP matrix of the propagated field can be expressed as

$$
\begin{aligned}
& J_{j k}\left(\mathbf{r}_{1}, \mathbf{r}_{2}, z\right)=\sum_{l, m=x}^{y} \frac{1}{\lambda^{2} z^{2}} \exp \left[\frac{\mathrm{i} \zeta}{2}\left(r_{2}^{2}-r_{1}^{2}\right)\right] \\
& \quad \times \iint T_{j l}\left(\boldsymbol{\rho}_{1}\right) T_{k m}^{*}\left(\boldsymbol{\rho}_{2}\right) J_{l m}^{\mathrm{in}}\left(\boldsymbol{\rho}_{1}, \boldsymbol{\rho}_{2}, 0\right) \\
& \quad \times \exp \left[\frac{\mathrm{i} \zeta}{2}\left(\rho_{2}^{2}-\rho_{1}^{2}-2 \mathbf{r}_{2} \cdot \boldsymbol{\rho}_{2}+2 \mathbf{r}_{1} \cdot \boldsymbol{\rho}_{1}\right)\right] d^{2} \rho_{1} d^{2} \rho_{2},
\end{aligned}
$$

where $\zeta=2 \pi /(\lambda z)$ and the dot represents the scalar product.

On the other hand, the free-space propagation of the unperturbed input field can be evaluated as

$$
J_{j k}^{\text {in }}\left(\mathbf{r}_{1}, \mathbf{r}_{2}, z\right)=\frac{\exp (\mathrm{i} \zeta \mathbf{s} \cdot \mathbf{t})}{\lambda^{2} z^{2}} H_{j k}(\mathbf{s}, \mathbf{t})
$$

where the auxiliary functions $H_{j k}(\mathbf{s}, \mathbf{t})$ are introduced as

$$
\begin{aligned}
& H_{j k}(\mathbf{s}, \mathbf{t})= \\
& \quad \iint J_{j k}^{\text {in }}\left(\boldsymbol{\rho}_{1}, \boldsymbol{\rho}_{2}, 0\right) \exp \left[\frac{\mathrm{i} \zeta}{2}\left(\rho_{2}^{2}-\rho_{1}^{2}\right)\right] \\
& \quad \times \exp \left[-\mathrm{i} \zeta\left(\mathbf{r}_{2} \cdot \boldsymbol{\rho}_{2}-\mathbf{r}_{1} \cdot \boldsymbol{\rho}_{1}\right)\right] d^{2} \rho_{1} d^{2} \rho_{2},
\end{aligned}
$$

being $\mathbf{s}=\left(\mathbf{r}_{1}+\mathbf{r}_{2}\right) / 2$ and $\mathbf{t}=\mathbf{r}_{2}-\mathbf{r}_{1}$. The explicit dependence of $H_{j k}$ on $z$ has been omitted for brevity.

Therefore, from Eq. (9), the BCP elements corresponding to the propagated field behind the optical element can be expressed as

$$
\begin{aligned}
J_{j k}\left(\mathbf{r}_{1}, \mathbf{r}_{2}, z\right) & =\frac{1}{\lambda^{2} z^{2}} \exp \left[\frac{\mathrm{i} \zeta}{2}\left(r_{2}^{2}-r_{1}^{2}\right)\right] \\
& \times \sum_{l, m=x}^{y} j_{j k}^{l m}\left(\mathbf{r}_{1}, \mathbf{r}_{2}, z\right),
\end{aligned}
$$

where the functions

$$
\begin{aligned}
j_{j k}^{l m}\left(\mathbf{r}_{1}, \mathbf{r}_{2}, z\right) & =\sum_{q, q^{\prime}} a_{j l}^{q}\left(a_{k m}^{q^{\prime}}\right)^{*} \\
\times & H_{l m}\left(\mathbf{s}-\frac{\gamma_{j l}^{q}}{2 \zeta}+\frac{\gamma_{k m}^{q^{\prime}}}{2 \zeta}, \mathbf{t}+\frac{\gamma_{j l}^{q}}{\zeta}+\frac{\gamma_{k m}^{q^{\prime}}}{\zeta}\right)
\end{aligned}
$$

are linear combinations of the auxiliary functions $H_{l m}(\mathbf{s}, \mathbf{t})$ and different tilted versions of them. The particular set of linear combinations and tilts depend on the specific optical element considered.

Then, for any PLDS, Eqs. (12) and (13) allow to calculate irradiance and polarization characteristics of the diffracted beam at any distance, if the free-space propagation of the incident beam is known.

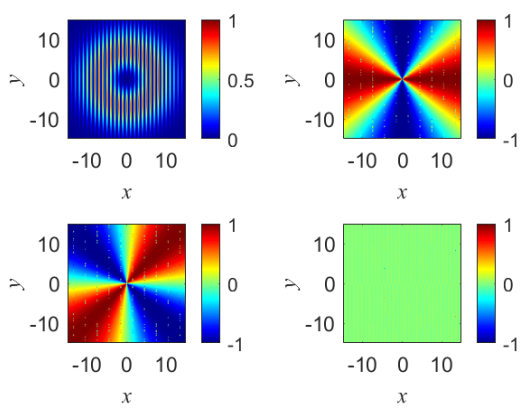

Figure 1. Intensity, normalized to its maximum, and normalized Stokes parameters, $S_{1}, S_{2}$, and $S_{3}$, from left to right and top to down, for a radially polarized partially coherent beam at the exit plane of a sinusoidal amplitude grating. Lenghts in $L_{x}$ units

\section{Example}

As a particular case, we consider a sinusoidal amplitude grating represented by a Jones matrix of the form

$$
\widehat{T}(\mathbf{r})=\frac{1}{2}[1+\cos (\gamma x)] \widehat{I},
$$

where $\widehat{I}$ is the $2 \mathrm{D}$ identity matrix and $\gamma=2 \pi / L_{x}$, with $L_{x}$ being the grating period.

As the input beam, we consider a radially polarized partially coherent beam, with Gaussian degree of coherence and donut-like intensity profile, described by the following BCP matrix [14]:

$$
\begin{aligned}
& \widehat{J}^{\text {in }}\left(\boldsymbol{\rho}_{1}, \boldsymbol{\rho}_{2}, 0\right)= \\
& \quad \frac{I_{0}}{4 \sigma^{2}} \exp \left[-\frac{\rho_{1}^{2}+\rho_{2}^{2}}{4 \sigma^{2}}-\frac{\left(\boldsymbol{\rho}_{1}-\boldsymbol{\rho}_{2}\right)^{2}}{2 \mu^{2}}\right]\left(\begin{array}{ll}
\xi_{1} \xi_{2} & \xi_{1} \eta_{2} \\
\eta_{1} \xi_{2} & \eta_{1} \eta_{2}
\end{array}\right) .
\end{aligned}
$$

Here, $I_{0}$ is a positive quantity with irradiance dimensions, $\sigma$ gives the spot size of the irradiance profile, and $\mu$ is the width of the degree of coherence at the $z=0$ plane.

Substitution of Eq. (15) into Eq. (9) leads to the BCP elements of the propagated field after the diffractive element. Irradiance, DoP and Stokes vector of the propagated field can be calculated by substituting the values of $J_{j k}\left(\mathbf{r}_{1}, \mathbf{r}_{2}, z\right)$ into Eqs. (2), (3) and (4) respectively. Since the resulting analytical expressions for such quantities are rather cumbersome, we omit them, and limit ourselves to show results obtained from them.

Figure 1 shows the Stokes parameters of the beam at the exit plane of the grating (with $\sigma=5 L_{x}$ ). Note that $S_{0}$ is proportional to the intensity. The DoP (not shown) is unitary everywhere at the exit plane, regardless the values of the input beam parameters $\sigma$ and $\mu$, and the state of polarization corresponds to that of a radially polarized beam. The only effect at the exit plane is the sinusoidal modulation of the intensity.

When the output beam propagates, its polarization state changes in different ways for diverse values of $\mu$. Figures 2 and 3 show the Stokes parameters of the beam of Fig. 1 at a propagation distance $z=2 L_{x}^{2} / \lambda$. 

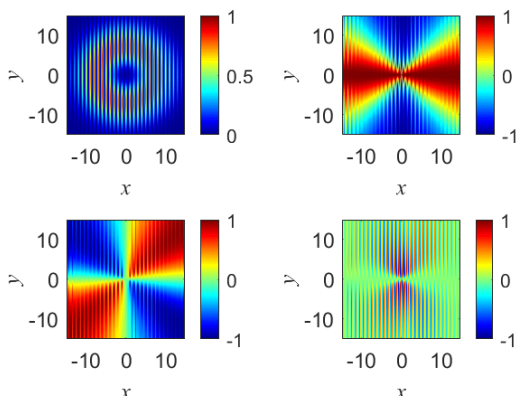

Figure 2. As Fig. 1, for $z=2 L_{x}^{2} / \lambda$ and $\mu=10^{6} \iota_{x}$.
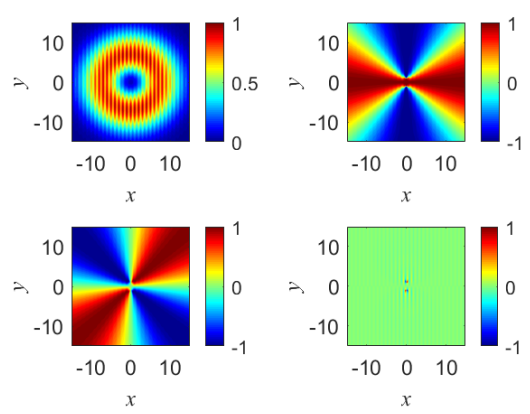

Figure 3. As Fig. 1, for $z=2 L_{x}^{2} / \lambda$ and $\mu=\mathrm{七}_{x}$.

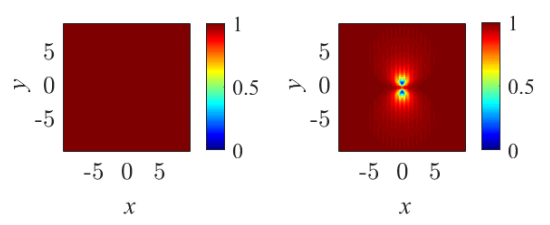

Figure 4. DoP for quasi coherent (left, $\mu=10^{6} \mathfrak{七}_{x}$ ) and partially coherent (right, $\mu=\mathrm{七}_{x}$ ) source at $z=2 L_{x}^{2} / \lambda$.

Figure 2 corresponds to a practically coherent beam $\left(\mu=10^{6} L_{x}\right)$. The DoP turns out to be unitary at any point across the transverse plane (see left part of Fig. 4), while the polarization state is no longer radially polarized, specially in those regions where the intensity is appreciably large. Note that the polarization characteristics of radially polarized perfectly coherent beams $(\mu \rightarrow \infty)$ remain invariant under propagation through homogeneous linear optical systems.

Figure 3 corresponds to a partially coherent input beam (with $\mu=L_{x}$ ). It can be noted that the fringe visibility of the intensity profile is lower than for the coherent case. Near the center of the beam, the polarization state is not radially polarized (compare with $S_{1}, S_{2}$, and $S_{3}$ in Fig. 1), and the DoP appreciably decreases in two vertical lobes (see right part of Fig. 4). However, outside the central area (the central area has low intensity), the DoP is nearly one and the polarization is approximately radial.

\section{Conclusion}

In this work, we study the behavior of a partially coherent and partially polarized beam when it passes through a periodic linear deterministic structure. The general case is developed for any beam with known free space propagation properties and for any periodic structure that can be represented by a Jones matrix. An example is presented for a particularly simple periodic structure (a sinusoidal amplitude grating) and a radially polarized input beam. Changes in the polarization characteristics with propagation are more evident for a coherent beam than for a partially coherent one.

\section{Acknowledgments}

This work has been partially supported by Spanish Ministerio de Economía y Competitividad under project FIS2013-46475.

\section{References}

[1] M. Born and E. Wolf, Principles of Optics. Cambridge University Press, Sixth (corrected) ed.

[2] D. H. Goldstein, Polarized Light. Marcel Dekker, Inc., Second (revised and expanded) ed.

[3] M. Dušek, "Diffraction of partially coherent beams on threedimensional periodic structures and the angular shifts of the diffraction maxima," Phys. Rev. E, vol. 52, pp. 6833-6840, Dec 1995.

[4] E. Wolf, "Diffraction of radiation of any state of spatial coherence on media with periodic structure," Opt. Lett., vol. 38, pp. 4023-4025, Oct 2013.

[5] M. Santarsiero, J. C. G. de Sande, G. Piquero, and F. Gori, "Coherencepolarization properties of fields radiated from transversely periodic electromagnetic sources," Journal of Optics, vol. 15, no. 5, p. 055701, 2013.

[6] J. C. G. de Sande, M. Santarsiero, G. Piquero, and F. Gori, "Longitudinal polarization periodicity of unpolarized light passing through a double wedge depolarizer," Opt. Express, vol. 20, pp. 27348-27360, Dec 2012.

[7] F. Gori, "Matrix treatment for partially polarized, partially coherent beams," Opt. Lett., vol. 23, pp. 241-243, Feb 1998.

[8] F. Gori, M. Santarsiero, S. Vicalvi, R. Borghi, and G. Guattari, "Beam coherence-polarization matrix," Pure and Applied Optics: Journal of the European Optical Society Part A, vol. 7, no. 5, p. 941, 1998.

[9] E. Wolf, Introduction to the Theory of Coherence and Polarization of Light. Cambridge University Press.

[10] R. Martínez-Herrero, P. M. Mejías, and G. Piquero, Characterization of Partially Polarized Light Fields. Springer Series in Optical Science, Springer.

[11] F. Gori, "Measuring Stokes parameters by means of a polarization grating," Opt. Lett., vol. 24, pp. 584-586, May 1999.

[12] J. C. G. de Sande, G. Piquero, M. Santarsiero, and F. Gori, "Partially coherent electromagnetic beams propagating through double-wedge depolarizers," Journal of Optics, vol. 16, no. 3, p. 035708, 2014.

[13] L. Mandel and E. Wolf, Optical Coherence and Quantum Optics. Cambridge University Press.

[14] G. Wu, F. Wang, and Y. Cai, "Coherence and polarization properties of a radially polarized beam with variable spatial coherence," Opt. Express, vol. 20, pp. 28301-28318, Dec 2012. 